\title{
Design of Slag Thickness Fuzzy Control System for Slag Adding Robot
}

\author{
Yuan GUO, Xiao-Hui ZHAN, Xin-Yuan* CHEN, Jiang-Hong DENG \\ College of Machinery and Automation, Wuhan University of Science and Technology, Wuhan, China \\ 323111116@qq.com,598258094@qq.com,13627293882@163.com,Dengjianghong325@163.com
}

\begin{abstract}
According to the defect of artificial slag adding and open-loop slag adding in continuous casting mold, a slag adding robot with real-time slag thickness detection and feedback control is developed. That is, the laser ranging sensor is applied on the basis of the open-loop slag adding robot. Then the real-time information of in-mold slag thickness can be obtained. And the coupling relation of three factors: real-time slag thickness, mold work slagging speed and robot slagging rate are taken into comprehensive consideration. Therefore fuzzy controller is built to realize the fuzzy PID control of the slagging robot feeding electro-mechanical system and achieve the intelligent control of slag thickness in the mold. The simulation and application results show that the slag adding robot based on fuzzy PID control has good effect and quick response. The slag can be pressed according to the requirement, which is beneficial to energy saving and consumption reduction, and improves the quality of the billet.
\end{abstract}

\section{Introduction}

In the process of continuous casting of steel, the thickness of the mold powder seriously affects the quality of billet [1-5]. Too great thickness of the mold fluxes would lead surface crack of billet to be increased and slag powder to be loosen. However, oxidation of molten steel, fast cooling and the wear of the mold will happen if the thickness is too small. Therefore, it is necessary to precisely control the thickness of the slag [6-9]. At present artificial slag adding is adopted in most enterprises without accuracy. A few enterprises use slag machine to replace artificial slag adding to achieve automation.But in this open-loop way the amount of slag added can't be real-time adaptively controlled with the change of the parameters. In this paper adding slag process of the mold fluxes is taken into account. By associating with the actual slag requirements a slag adding robot is developed. Through the laser distance measuring sensor, the thickness of the mold fluxes is real-time scanned and measured, and feedback control can be realized to adjust the amount of slag dropping accurately so as to realize the accurate control of the thickness of the mold fluxes in the mold[10-13].

\section{System of slag thickness adaptive slag adding robot}

\subsection{Open-loop System of Slag Machine}

The slag adding robot of continuous casting mold developed by our research group, which consists of electronic control system (1), movement system(2), adding slag system (3), and Lifting system (4).The adding slag system is made up of hopper 1 , gear reducer 2 and 4 , transporting motor 3, swinging-rod motor 5, transporting pipe 6 , guiding device 7 , transporting spiral 8 , discharging port 9 and so on. The structure of the slag adding robot is shown in Fig.1.

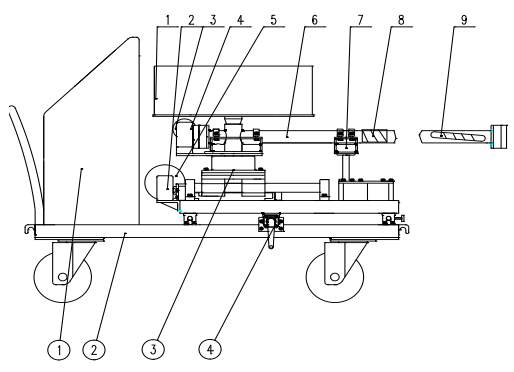

Fig.1 The structure of the slag adding robot

The mold fluxes is fed from the hopper into the feed pipe, and the feed motor drives the feed screw to deliver the slag to the discharge port, then the mold fluxes falls into the mold. The swing bar motor drives the feed pipe to swing slag in the slag adding area of the mold. Fig. 2 is a diagram of open-loop slag adding. The single tube distribution range is $1300 \mathrm{~mm} * 250 \mathrm{~mm}$, the single pipe slag flow range is from $0.15 \mathrm{~L} / \mathrm{min}$ to $1.5 \mathrm{~L} / \mathrm{min}$ and the feeding cycle is from $6 \mathrm{~s}$ to $60 \mathrm{~s}$. 


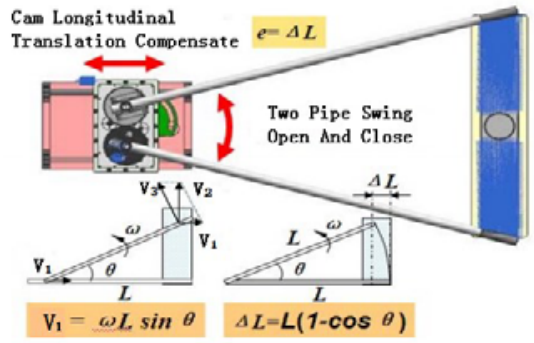

Fig. 2 The diagram of slag adding for the open-loop slag adding robot

\subsection{System of Closed-loop Slag Adding Robot}

Based on the developed open-loop slag adding robot, the laser distance measuring sensor (as shown in Fig. 3) is added to the discharge port of the feed pipe to measure the thickness of mold fluxes[14-18]. In order to avoid the influence of the color of molten steel on the measurement results, a green laser with different wavelength range was selected. By using the real-time slag thickness measured through the laser distance measuring sensor as a feedback signal, the closed-loop control of slag adding robot is realized.

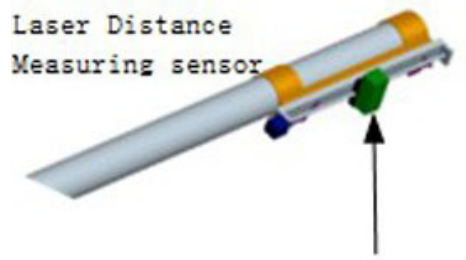

Fig. 3 Laser scanning device for slag thickness

\subsection{Control Model of the Slag Adding}

In the process of adding slag, the instructions are transferred from the computer to the inverter firstly, and then the inverter transfers the received signal to the feeding motor, a feeding screw driven by the feeding motor drives the mold fluxes to the discharge port so as to add the mold fluxes into the crystallizer. The components of the slag adding process are connected in series, and the transfer function of each link is calculated. The inverter can be simplified into an inertial link [19-20] in the process of adding slag, that is, the transfer function of the converter is:

$$
G_{1}(S)=\frac{K_{1}}{1+T_{1} S}
$$

The coefficient of proportionality $K_{1}$ of the inverter is 5 , and $T_{1}$ is the time constant of the inertial link, it usually tens to hundreds of $\mathrm{ms}$, and the value of $T_{1}$ is 0.3 . In the process of adding slag, the feeding motor can be simplified as an inertial link, that is, the transfer function of the feeding motor is:

$$
G_{2}(S)=\frac{K_{2}}{1+T_{2} S}
$$

In the formula, $K 2$ is the transmission coefficient of the feeding motor, and $T_{2}$ is the electromechanical time constant of the dragging system for the feeding motor, and $K_{2}$ is $0.2124 ; T_{2}$ is 0.075 according to the selected feeding motor.

The reducer and feed screw can be equivalent to a proportional link in the process of slag adding, the coefficient of proportionality is $K_{3}$, and the value is 0.133 . The open-loop transfer function of the slag adding robot is:

$$
G(S)=\frac{K_{1} K_{2} K_{3}}{\left(1+T_{1} S\right)\left(1+T_{2} S\right)}
$$

The open-loop transfer function of the slag adding robot can be calculated by substituting the specific value in formula (3):

$$
G(S)=\frac{0.1416}{(1+0.075 S)(1+0.3 S)}
$$

The thickness of adding slag fed back from the laser distance measuring sensor is fed to the computer, and the closed-loop control of slag adding capacity of the slag adding robot can be realized. The closed-loop control flow of the slag adding robot is shown in fig. 4 .

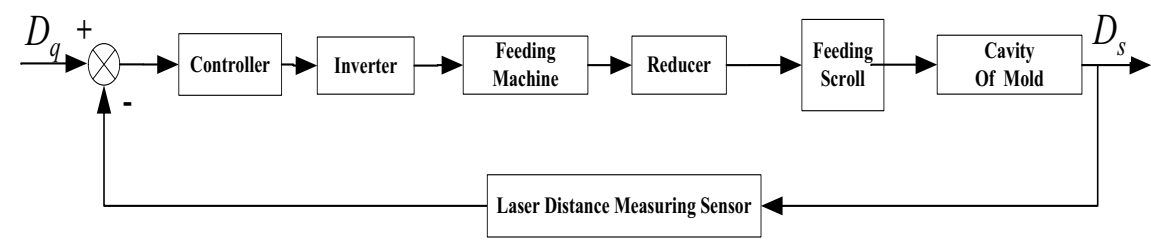

Fig. 4 Closed- loop control flow chart of adding slag robot

The slag is added to the mold as needed, and the amount of slag added is expected to be as follows:

$$
D_{q}=h-D_{c}+D_{x}
$$

In the formula, $D_{\mathrm{q}}$ is the expected thickness of the adding slag, $h$ is the best thickness of mold fluxes and its range is $35 \mathrm{~mm} \sim 50 \mathrm{~mm}, D_{\mathrm{c}}$ is the thickness of the slag measured by the laser distance measuring sensor, and $D_{\mathrm{x}}$ is the thickness consumed by the mold in unit time. And the expected amount of the adding slag is:

$Q=\frac{D_{q} l x}{t}$

In the formula, $l$ is the width of the mold, $x$ is the length the mold, and $t$ is the unit time. 


\section{Design of the controller}

In the thickness control system of the adding slag robot, the controller is the core of the system. During the crystallization period, the thickness of the mold fluxes should be $35 \mathrm{~mm}-50 \mathrm{~mm}$, the conventional PID controller often has poor parameter setting and poor adaptive ability [8-9], so the adaptive fuzzy PID control is adopted.

The structure of the fuzzy PID controller is shown in Fig.5, and the parameters of the PID are adjusted by fuzzy control. Set the deviation $e$ and deviation change rate $e c$ as input signal of the fuzzy controller, the fuzzy $E$ and $E C$ were obtained through fuzzy inference, self-tuning parameters such as $\Delta k_{\mathrm{p}} 、 \Delta k_{\mathrm{i}}$ and $\Delta k_{\mathrm{d}}$ of the fuzzy PID can be obtained by the fuzzy inference, $y d$ is the expected amount of the adding slag, $D s(t)$ is the actual thickness of the adding slag. The adjustment process of fuzzy PID parameters is to obtain the self-tuning values of three parameters of PID by fuzzy inference and constantly check and correct in the process of adding slag. The adjustment of the fuzzy PID parameters is as follows:

$\left\{\begin{array}{c}k_{p}=k_{p}^{\prime}+\Delta k_{p} \\ k_{i}=k_{i}^{\prime}+\Delta k_{i} \\ k_{d}=k_{d}^{\prime}+\Delta k_{d}\end{array}\right.$

In the formula, the initial parameters of the PID controller are $k_{p}^{\prime}, k_{\mathrm{I}}^{\prime}$ and $k_{\mathrm{d}}^{\prime}$

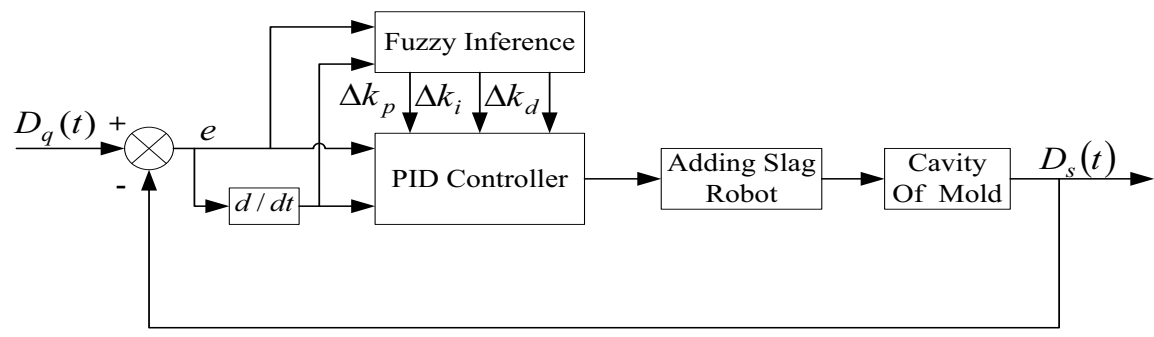

Fig. 5 Structure diagram of fuzzy controller

Determination of initial parameters of PID: Z-N method is used to obtain initial parameters of PID controller. In the control process, the Z-N frequency domain tuning method is based on the stability analysis of the frequency domain response PID tuning method. The main idea of the method is: setting for the transfer function of the control system of the slag thickness, which the root locus can be obtained. The point through the $\mathrm{j} \omega$ axis, the corresponding gain is $\mathrm{Km}$, the corresponding values for $\omega$ is $\omega \mathrm{m}$ [20-25]. The formula is as follows:

$$
\begin{aligned}
& k_{p}=0.6 k_{m} \\
& k_{d}=\frac{k_{p} \pi}{4 \omega_{m}} \\
& k_{i}=\frac{k_{p} \omega_{m}}{\pi}
\end{aligned}
$$

In the formula, $\mathrm{km}$ is the gain value of the $\mathrm{K}$ when the system starts oscillation, and the $\omega \mathrm{m}$ is the oscillation frequency.

Between the actual output quantity and the desired output quantity of the crystallization slag thickness protector control system, the deviation e and the deviation change rate ec are the input quantity, and the three change value of the parameters $(\Delta \mathrm{kp} 、 \Delta \mathrm{ki}$ and $\Delta \mathrm{kd})$ of fuzzy PID are the output quantity. The mold fluxes thickness of the crystallizer is in the range of $35 \mathrm{~mm} \sim 50 \mathrm{~mm}$, the domain of the basic theory of the deviation e is [-7.5,7.5], the basic domain error rate is $[-5,5]$, the three basic domain of the PID are $\Delta \mathrm{kp} \in[-0.3,0.3], \Delta \mathrm{ki} \in[-0.06,0.06]$ and $\Delta \mathrm{kd}$ $\in[-6,6]$.

In the selection of the membership function, generally the Gauss type and the triangle type are used, the Gauss type is used for the boundary part of the deviation $e$ and the deviation change rate $e c$, while the triangle type is used for the PID three tuning parameters and the other parts except the deviation $e$ and deviation change rate $e c$ because the calculation of the triangle type is simple. The slag quantity deviation $e$, the deviation change rate $e c$, the PID self-tuning parameter $\Delta k_{\mathrm{p}}, \Delta k_{\mathrm{i}}$ and $\Delta k_{\mathrm{d}}$ are all set as $\{N B, M N, N S, Z O, P S, P M, P B\}$. They respectively represent the negative big, negative middle, negative small, zero, positive small, positive middle and positive large. The fuzzy control rule table is shown in table 1 . 
Table 1 Fuzzy control rules tables of the $\Delta k_{p} 、 \Delta k_{i}$ and $\Delta k_{d}$

\begin{tabular}{c|c|c|c|c|c|c|c|c}
\hline \multirow{2}{*}{$\Delta k_{p} 、 k_{i}, \Delta k_{d}$} & \multicolumn{7}{|c}{$E C$} \\
\cline { 3 - 9 } & $N B$ & $N B / N B / P S$ & $P B / N B / N S$ & $P M / N M / N B$ & $P M / N M / N B$ & $P S / N S / N B$ & $Z O / Z O / N M$ & $Z O / Z O / P S$ \\
\hline \multirow{5}{*}{$E$} & $N M$ & $P B / N B / P S$ & $P B / N B / N S$ & $P M / N M / N B$ & $P S / N S / N M$ & $P S / N S / N M$ & $Z O / Z O / N S$ & $N S / Z O / Z O$ \\
\cline { 2 - 9 } & $N S$ & $P M / N B / Z O$ & $P M / N M / N S$ & $P M / N S / N M$ & $P S / N S / N M$ & $Z O / Z O / N S$ & $N S / P S / N S$ & $N S / P S / Z O$ \\
\cline { 2 - 9 } & $Z O$ & $P M / N M / Z O$ & $P M / N M / N S$ & $P S / N S / N S$ & $Z O / Z O / N S$ & $N S / P S / N S$ & $N M / P M / N S$ & $N M / P M / Z O$ \\
\cline { 2 - 9 } & $P S$ & $P S / N M / Z O$ & $P S / N S / Z O$ & $Z O / Z O / Z O$ & $N S / P S / Z O$ & $N S / P S / Z O$ & $N M / P M / Z O$ & $N M / P B / Z O$ \\
\cline { 2 - 9 } & $P M$ & $P S / Z O / P B$ & $Z O / Z O / N S$ & $N S / P S / P S$ & $N M / P S / P S$ & $N M / P M / P S$ & $N M / P B / P S$ & $N B / P B / P B$ \\
\cline { 2 - 9 } & $P B$ & $Z O / Z O / P B$ & $Z O / Z O / P M$ & $N M / P S / P M$ & $N M / P M / P M$ & $N M / P M / P S$ & $N B / P B / P S$ & $N B / P B / P B$ \\
\hline
\end{tabular}

The method of solving fuzzy rules in this paper is the gravity method, the formula is as follows:

$u_{0}=\frac{\int_{v} u \mu(u) \mathrm{d} u}{\int_{v} \mu(u) \mathrm{d} u}$

In the formula: $u$ is the element of output domain, $u_{0}$ is the result of defuzzification, and $\mu(u)$ is the membership function.

In the course of adjustment of PID fuzzy parameters, when the thickness of the slag in the mold gets smaller, we should increase the amount of slag added to the system, increase the value of $k_{\mathrm{p}}$, and take the larger $k_{\mathrm{p}}$. However, in order to avoid differential supersaturation we should take smaller $k_{\mathrm{d}}$ and take $k_{\mathrm{i}}=0$ in order to avoid over retrieval. When the slag thickness is moderate, the effect of $\mathrm{kd}$ on the system is great, so the value of $k_{\mathrm{d}}$ should be adjusted reasonably.

\section{Fuzzy PID Control And Simulation}

Fuzzy PID control model was built by the Matlab/Simulink toolbox, and simulation analysis is carried out as well. The three initial parameters of the PID controller are: $\mathrm{kp}=114, \mathrm{ki}=1.6, \mathrm{kd}=74$, step signals and slag adding are respectively used as the input signals for the simulation analysis. The simulation results are shown in Fig. 6 and Fig. 7. The horizontal axis in Fig. 6 is time, and Fig. 7 is a fuzzy PID control diagram for controlling the side of a mold during a slag adding cycle.

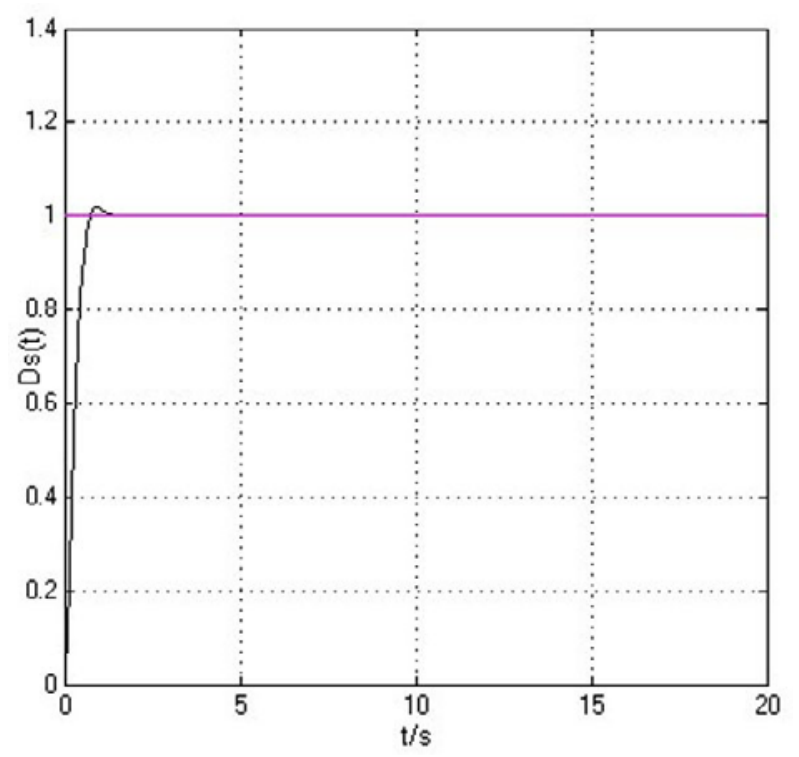

Fig. 6 Response of step signal

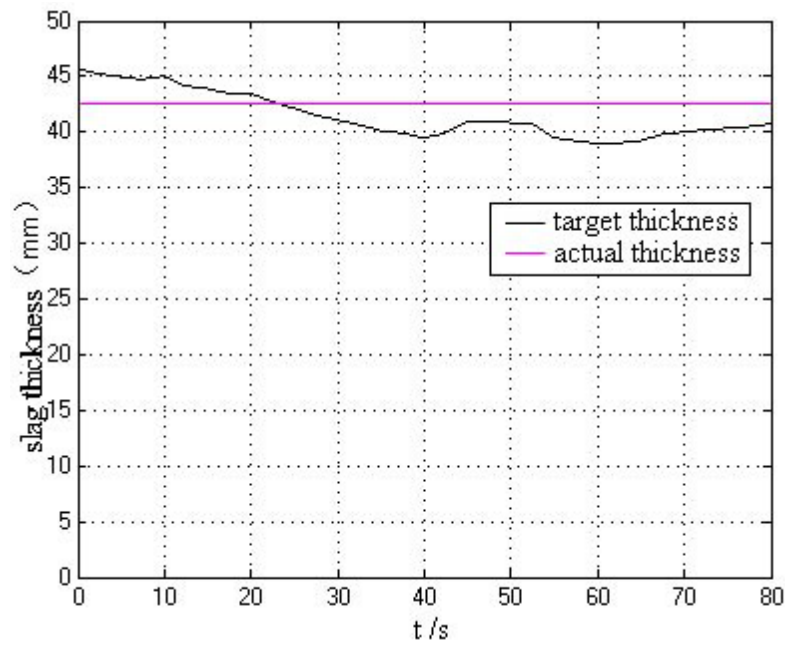

Fig. 7 Fuzzy PID control effect diagram

It can be seen in Fig. 6 that the system has fast response speed and high control precision. Fig. 7 showed that the mold fluxes thickness of the PID fuzzy control system of the crystallizer focus between the $35 \mathrm{~mm}-50 \mathrm{~mm}$, which meets the control requirements, and the control effect of the system is good. 


\section{Field Service}

The equipment developed by the research group has been used online for about 2 years in the 4th steel mill of Wuhan Iron and Steel (Group) Corp. The result shows that the equipment can reduce the slag consumption efficiently and is conducive to the quality and stability of the products. The thickness of the slag ranges from $35 \mathrm{~mm}$ to $49 \mathrm{~mm}$. The picture of the field service is shown in fig. 8 .

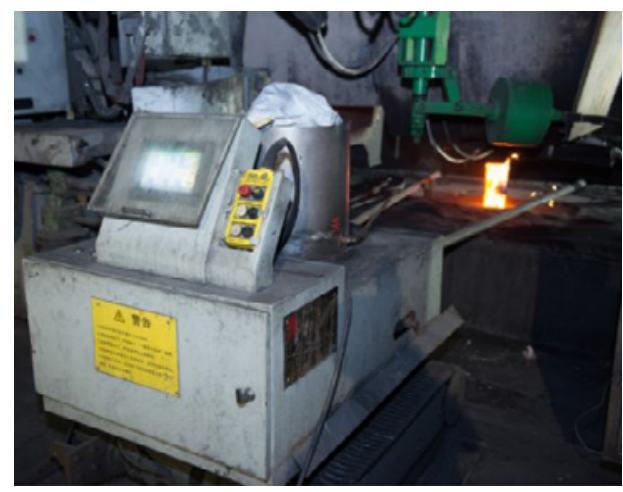

Fig.8 Online application scheme of fuzzy control slag adding robot in the 4th steel mill ofWuhan Iron and Steel (Group) Corp.

\section{Conclusion}

In this paper, the laser distance measuring sensor is used as feedback device in order to meet the requirement of slag adding process. The fuzzy controller has been developed by adopting adaptive fuzzy PID algorithm. And the slag adding robot with closed- loop system could achieve real-time adjustment as required. So the slag adding robot can adapt to the change of continuous casting crystallization process parameters and realize slag adding on demands. The field application shows that the control system of this equipment has good effect on stability and control accuracy.

\section{Acknowledgement}

Fund project: Supported by Hubei science and technology support plan project (2015BAA023)

Project supported by key technological research project of Wuhan (2016010101010011)

\section{References}

[1]Effect of casting powder on casting blank Quality and Continuous Casting Process [J] LYS Science-Technology \& Management, 2009, 06:61.

[2] Man-tang DING. Research of Fluoride-free Protective Slag Used in low C Steel. Advanced Materials Research Vol 803, 2013: 312-316

[3] Continuous Casting Technology question-answering [M]. First edition. Beijing: Chemical Industry Press, 2013:273-300.

[4]Zhong Yuntao, Liqiang. Application of Mold Power in Continuous casting machine. $[\mathrm{J}]$ Continuous
Casting, 2013 04:9-13.

[5]Memon I, Chen L, Majid A, et al. Travel Recommendation Using Geo-tagged Photos in Social Media for Tourist [J]. Wireless Personal Communications, 2015, 80(4): 1347-62.

[6]Memon I. Authentication user's privacy: An integrating location privacy protection algorithm for secure moving objects in location based services $[\mathrm{J}]$. Wireless Personal Communications, 2015, 82(3): 1585-1600.

[7]Arain Q A, Memon H, Memon I, et al. Intelligent travel information platform based on location base services to predict user travel behavior from user-generated GPS traces[J]. International Journal of Computers and Applications, 2017: 1-14.

[8]Memon I, Arain Q A. Dynamic path privacy protection framework for continuous query service over road networks[J]. World Wide Web, 2017, 20(4): 639-672.

[9]Arain Q A, Memon I, Deng Z, et al. Location monitoring approach: multiple mix-zones with location privacy protection based on traffic flow over road networks[J]. Multimedia Tools and Applications, 2017: 1-45.

[10]Memon I. A secure and efficient communication scheme with authenticated key establishment protocol for road networks[J]. Wireless Personal Communications, 2015, 85(3): 1167-1191.

[11]Arain Q A, Zhongliang D, Memon I, et al. Privacy Preserving Dynamic Pseudonym-Based Multiple Mix-Zones Authentication Protocol over Road Networks[J]. Wireless Personal Communications, 1-17.

[12]Arain Q A, Uqaili M A, Deng Z, et al. Clustering Based Energy Efficient and Communication Protocol for Multiple Mix-Zones Over Road Networks[J]. Wireless Personal Communications, 1-18.

[13]Memon I, Arain Q A. Optimal placement of mix zones in road networks[J]. arXiv preprint arXiv:1705.11104, 2017.

[14]Memon I, Arain Q A, Memon M H, et al. Search me if you can: Multiple mix zones with location privacy protection for mapping services[J]. International Journal of Communication Systems, 2017.

[15]Mangi F A, Xiao S, Jamro D A, et al. Manipulating Electromagnetic Wave Linear-to-Circular Polarization Conversion Transmitter Based on Periodic Strips Array[C]//Information Science and Control Engineering (ICISCE), 2016 3rd International Conference on. IEEE, 2016: 1342-1345.

[16]Memon M H, Li J P, Memon I, et al. Efficient object identification and multiple regions of interest using CBIR based on relative locations and matching regions[C]//Wavelet Active Media Technology and Information Processing (ICCWAMTIP), 2015 12th International Computer Conference on. IEEE, 2015: 247-250.

[17]Memon M H, Khan A, Li J P, et al. Content based image retrieval based on geo-location driven image tagging on the social web[C]//Wavelet Active Media Technology and Information Processing (ICCWAMTIP), 2014 11th International Computer 
Conference on. IEEE, 2014: 280-283.

[18]Memon I, Mangi F A, Jamro D A. Collision avoidance of intelligent service robot for industrial security system[J]. IJCSI International Journal of Computer Science Issues, 2013, 10(3).

[19]Kamenyi D M, Wang Y, Zhang F, et al. Authenticated privacy preserving for continuous query in location based services[J]. Journal of Computational Information Systems, 2013, 9(24): 9857-9864.

[20]SuZhiwei, ChenXinyuan, DengJianghong, Zhan Xiaohui. Design of casting power adding area rectangular compensation mechanism for casting power feeder of continuous casting crystallizer. [J] Chinese Journal of Engineering Design, 2015 05:420-424.

[21]Chen Xinyuan, SuZhiwei, Zhan Xiaohui. A kind of wide slab mold with double pipe automatic slag machine. [P].CN201510174589.1, 20150515.

[22]PengAnhong, Transmitting Function of lnverter Induction Motor[J], Anshan Institute of I \& S.technology, 200006 447-449.

[23]AL-GHAMDI A S.Performance Analysis of Automated Control System for Condenser Water Treatment Unit [J]. Engineering, 2012, 4(1):35-39

[24]Chen Lifu, Pan Gongyu, Zhang Qingqing. Self-adaptive Fuzzy-PID controller of pneumatic active suspension $\quad[\mathrm{J}]$. Machinery Design\&Manufacture. 2010 (08).

[25]Liu Jinkun. Advanced PID Control and MATLAB Simulation. [M] Beijing: Electronic Industry Press. 2010:77-87. 